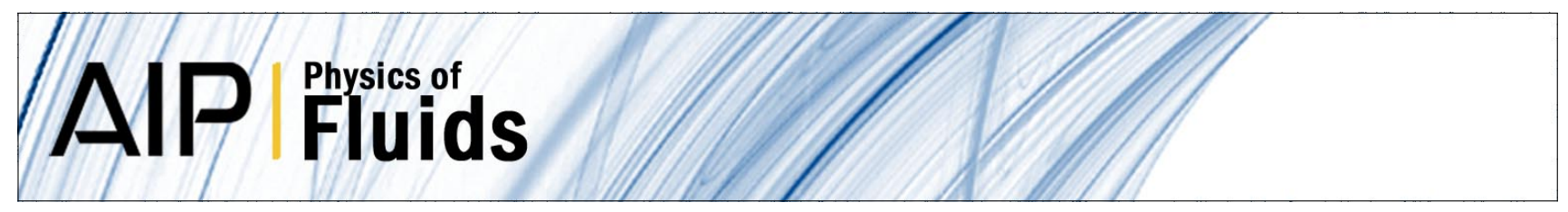

\title{
Inclusion of lubrication forces in dynamic simulations
}

Ashok S. Sangani and Guobiao Mo

Citation: Phys. Fluids 6, 1653 (1994); doi: 10.1063/1.868228

View online: http://dx.doi.org/10.1063/1.868228

View Table of Contents: http://pof.aip.org/resource/1/PHFLE6/v6/i5

Published by the American Institute of Physics.

\section{Related Articles}

Criticality of flow transition behind two side-by-side elliptic cylinders

Phys. Fluids 24, 034102 (2012)

Frictional slip lengths for unidirectional superhydrophobic grooved surfaces

Phys. Fluids 23, 072001 (2011)

A study of start-up flow of thixotropic fluids including inertia effects on an inclined plane

Phys. Fluids 23, 013103 (2011)

A formula for the wall-amplified added mass coefficient for a solid sphere in normal approach to a wall and its application for such motion at low Reynolds number

Phys. Fluids 22, 123303 (2010)

Axial pressure-difference between far-fields across a sphere in viscous flow bounded by a cylinder

Phys. Fluids 22, 103305 (2010)

\section{Additional information on Phys. Fluids}

Journal Homepage: http://pof.aip.org/

Journal Information: http://pof.aip.org/about/about_the_journal

Top downloads: http://pof.aip.org/features/most_downloaded

Information for Authors: http://pof.aip.org/authors

\section{ADVERTISEMENT}

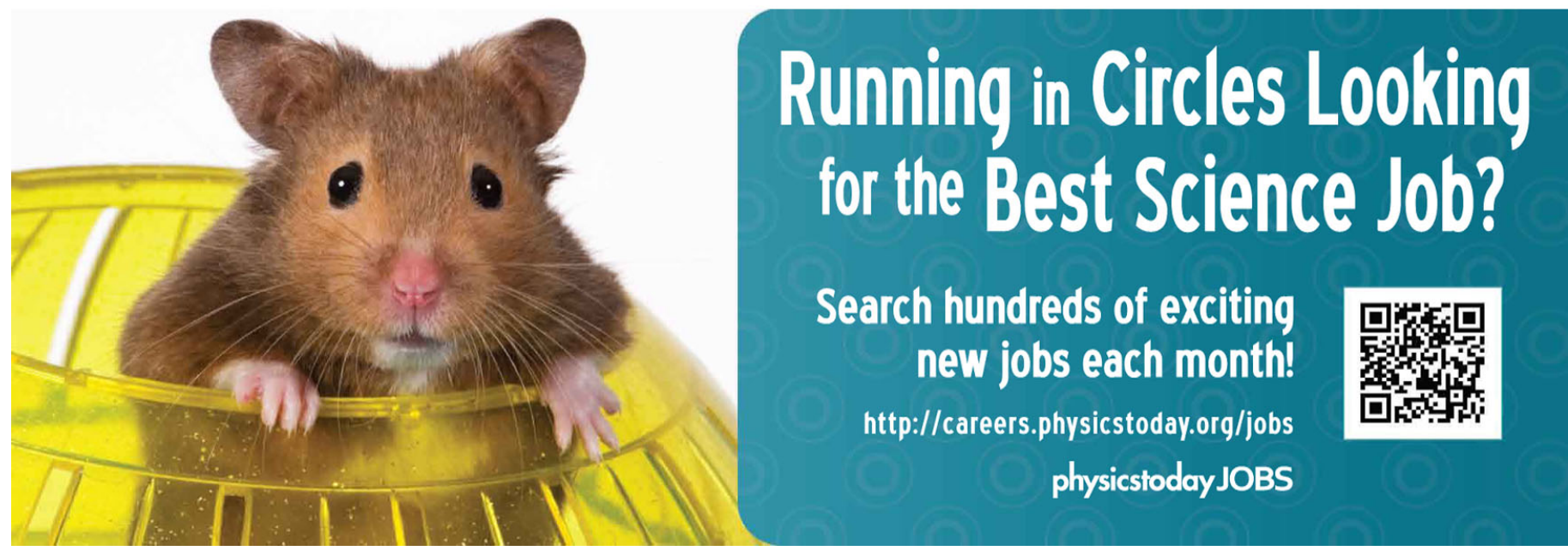




\title{
Inclusion of lubrication forces in dynamic simulations
}

\author{
Ashok S. Sangani and Guobiao Mo \\ Department of Chemical Engineering and Materials Science, Syracuse University, Syracuse, \\ New York 13244
}

(Received 1 October 1993; accepted 29 December 1993)

\begin{abstract}
A new method is described for incorporating close-field, lubrication forces between pairs of particles into the multiparticle Stokes flow calculations. The method is applied to the suspensions of both spherical as well as cylindrical particles, and results computed by the method are shown to be in excellent agreement with the exact known results available in the literature.
\end{abstract}

\section{INTRODUCTION}

In a companion paper, ${ }^{1}$ referred to henceforth as I, we presented details of a method for computing Stokes flow interactions among many particles. As mentioned in that paper, one of the drawbacks of the method is its inadequacy in treating problems in which the lubrication forces between the pair of particles in near contact play a dominant role. This difficulty was overcome by Brady and Bossis $^{2}$ who constructed an approximation to the manyparticle resistivity tensor by first obtaining a far-field approximation to the many-particle mobility tensor with a finite $N_{s}$ and then adding near-field interactions to the inverted mobility matrix. Here, $N_{s}-1$ refers to the highest force moment retained in the far-field approximation (cf. I). Their method was also employed by Ladd, $^{3}$ who showed that this approximation can be systematically improved by increasing $N_{s}$. The computationally intensive step in this method for large $N$, the number of interacting particles, is the inversion of the mobility matrix of size $N_{t}=\left(3 N_{s}^{2}-1\right) N$, which requires roughly $O\left(N_{t}^{3}\right)$ computations.

We present here an alternate scheme for incorporating the lubrication forces. It may be recalled that the method of multipole expansion replaces the actual force density distribution on the surface of a particle by a distribution of force and higher-order multipoles at the center of the particle. As two particles with finite relative motion approach each other, the force density in the region of narrow gap between the two particles rises, and one would need a large number of multipoles at the center of the particles to account for this singular nature of forces in the gap. Indeed, in some instances the method of multipole expansion may not converge at all with increasing $N_{s}$. To remedy this situation, it seems more reasonable to retain the singular nature of forces near the gap of the particles and expand only the remainder of the force density in multipoles at the center of the particles. The present study investigates in detail this alternate strategy and illustrates its accuracy for relatively small $N_{s}$.

The method can also be applied to other geometries of the particles or to governing equations other than the Stokes equations. As an example, we consider an interesting case of interactions among infinitely long cylinders in which the lubrication effects arise not only from the relative motion between the particles but also from a large pressure drop that occurs as a finite amount of fluid flows through a narrow gap between the particles. This problem is also important in the modeling of the diffusion of proteins in bilipid cell membranes. ${ }^{47}$

\section{THE METHOD FOR SPHERICAL PARTICLES}

As in $\mathrm{I}$, let us consider the problem of Stokes flow interactions among $N$ randomly placed spherical particles within a unit cell of a periodic array. For simplicity, we consider only the case of equisized rigid spheres. The velocity of the fluid is given by the integral equation ${ }^{8,9}$

$$
u_{i}(\mathbf{x})=U_{i}^{\infty}(\mathbf{x})-\frac{1}{4 \pi \eta} \sum_{\alpha=1}^{N} \int_{S^{\alpha}} f_{j}(\mathbf{y}) v_{i j}(\mathbf{x}-\mathbf{y}) d A_{\mathbf{y}},
$$

where $\eta$ is the viscosity of the fluid, $U_{i}^{\infty}(\mathbf{x})$ the macroscopic velocity, $f_{j}$ the force per unit area exerted by the fluid at a point $y$ on the surface $S^{\alpha}$ of the particle $\alpha$, and $v_{i j}$ the spatially periodic Green's function for the Stokes equations of motion [cf. (2) in I]. In the method of multipole expansion, $v_{i j}(\mathbf{x}-\mathbf{y})$ is expanded in a Taylor series near $v_{i j}\left(\mathbf{x}-\mathbf{x}^{\alpha}\right)$, and the resulting expression is integrated termwise to recast (1) to (4) in I. To account for the singular nature of lubrication forces between a pair of particles in near contact, we write $f_{i}=\hat{f}_{i}+f_{i}^{\text {lub }}$, and express only the contribution due to $\hat{f}_{i}$ in a multipole expansion. Thus, we write

$$
u_{i}(\mathbf{x})=U_{i}^{\infty}(\mathbf{x})+\sum_{\alpha=1}^{N} \mathscr{H}_{j}^{\alpha} v_{i j}\left(\mathbf{x}-\mathbf{x}^{\alpha}\right)+u_{i}^{\mathrm{lub}}(\mathbf{x}),
$$

where

$$
u_{i}^{\mathrm{lub}}(\mathbf{x})=-\frac{1}{4 \pi \eta} \sum_{\alpha=1}^{N} \int_{S^{\alpha}} f_{j}^{\mathrm{lub}}(\mathbf{y}) v_{i j}(\mathbf{x}-\mathbf{y}) d A_{\mathbf{y}},
$$

and

$$
\begin{aligned}
\mathscr{M}_{j}^{\alpha} v_{i j}\left(\mathbf{x}-\mathbf{x}^{\alpha}\right) \equiv & -\frac{1}{4 \pi \eta}\left[v_{i j}\left(\mathbf{x}-\mathbf{x}^{\alpha}\right) \int_{S^{\alpha}} \hat{f}_{j}(\mathbf{y}) d A_{\mathbf{y}}\right. \\
& -\partial_{k} v_{i j}\left(\mathbf{x}-\mathbf{x}^{\alpha}\right) \int_{S^{\alpha}} \hat{f}_{j}\left(y_{k}-x_{k}^{\alpha}\right) d A_{\mathbf{y}} \\
& +\cdots]
\end{aligned}
$$




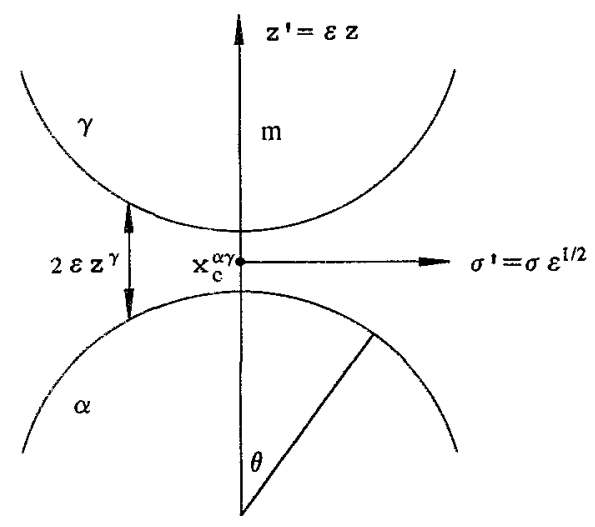

FIG. 1. Nomenclature for the analysis of the flow in the gap between two spheres.

The lubrication force density $\mathrm{f}^{\mathrm{lub}}$ in (3) must be determined as a function of the relative motion of the two particles through appropriate asymptotic analysis as will be done later in this article. In determining the strength of multipoles used in defining the differential operator $\mathscr{M}_{j}^{\alpha}$, we shall need to evaluate the contribution from $u_{i}^{\text {lub }}$ to various terms in the Lamb's general solution around each of the particles in the suspension. This, however, is computationally expensive owing to the integration required by (3). Since $f_{i}^{\text {lub }}$ will be chosen to be nonzero only in a small region near the gap between the particles, we can approximate (3) by the first two terms in the Taylor series expansion

$$
\begin{aligned}
\int_{S^{\alpha}} f_{j}^{\mathrm{lub}}(\mathbf{y}) v_{i j}(\mathbf{x}-\mathbf{y}) d A_{\mathbf{y}} \\
=v_{i j}\left(\mathbf{x}-\mathbf{x}_{c}^{\alpha \gamma}\right) \int_{S^{\alpha}} f_{j}^{\mathrm{lub}}(\mathbf{y}) d A_{\mathbf{y}}+\partial_{k} v_{i j}\left(\mathbf{x}-\mathbf{x}_{c}^{\alpha \gamma}\right) \\
\quad \times \int_{S^{\alpha}}\left(\mathbf{x}_{c}^{\alpha \gamma}-\mathbf{y}\right)_{k} f_{j}^{\mathrm{lub}}(\mathbf{y}) d A_{\mathbf{y}}+\cdots,
\end{aligned}
$$

where $\mathbf{x}_{c}^{\alpha \gamma}$ is midway through the gap between particles $\alpha$ and $\gamma$ in close proximity (cf. Fig. 1). If $\theta_{0}$ is the angle over which the lubrication force density is taken to be nonzero, then the error in truncating the above expression is $O\left(\theta_{0}^{2}\right)$ in the limit $\epsilon \rightarrow 0$.

The lubrication force on particle $\alpha$ will be chosen to be equal in magnitude but opposite in direction to that on particle $\gamma$ so that the contribution from the first term on the right-hand side of the above equation will vanish, and the lubrication velocity will consequently be approximated as

$$
u_{i}^{\mathrm{hub}}(\mathbf{x}) \approx \sum A_{j k}^{\alpha \gamma} \partial_{k} v_{i j}\left(\mathbf{x}-\mathbf{x}_{c}^{\alpha \gamma}\right),
$$

where the summation is over all the pairs whose separation distance is less than a specified value and

$$
A_{j k}^{\alpha \gamma}=\frac{1}{4 \pi \eta} \int_{S^{\alpha}+S^{\gamma}}\left(\mathbf{y}-\mathbf{x}_{c}^{\alpha \gamma}\right)_{k} f_{j}^{\mathrm{lub}}(\mathbf{y}) d A_{\mathbf{y}} .
$$

The lubrication velocity given by (6) is expected to be accurate provided that $\mathbf{x}$ is at a large distance from the gap between the particles and provided that the area over which lubrication force density is nonzero is small. Now in solving for the strengths of multipoles used in the expansion of the differential operator $\mathscr{M}_{j}^{\alpha}$ [cf. (5) in I], we need to evaluate derivatives of $u_{i}$, and hence $u_{i}^{\text {lub }}$, at the center $\mathbf{x}^{k}$ of a particle $k$. Here, $k$ may be any one of the $N$ particles in the cell including particles $\alpha$ and $\gamma$. Since $\left|\mathbf{x}^{k}-\mathbf{x}_{c}^{\alpha \gamma}\right|$ is large compared to the gap width, over which the lubrication force density is nonzero, the approximation to lubrication velocity as given by (6) is adequate for determining the multipoles associated with each particle in the suspension. It can be proved that this applies even to particles $\alpha$ and $\gamma$. The proof rests on the observation that, while the integrand in ( 3 ) is singular at $\mathbf{x}=\mathbf{y}, u_{i}^{\text {lub }}$ itself is continuous at the surface of particle $\alpha$. Thus, for the purpose of satisfying the boundary conditions on that particle, we first move the singularities at $S^{\alpha}$ radially outward from the surface by a small distance $\delta$. This renders the lubrication velocity and all its derivatives continuous for $\left|\mathbf{x}-\mathbf{x}^{\alpha}\right|<1+\delta$, the radius of the particle being unity. Therefore, it is possible to expand the lubrication velocity field in regular terms of the Lamb's solution [cf.(6) in I] and to satisfy the no-slip boundary conditions at $S^{\alpha}$ as if the lubrication singularities were all outside particle $\alpha$. The limit $\delta \rightarrow 0$ can subsequently be taken to show that this would yield results identical to that obtained by directly using (6). In other words, even though approximation (5) is not valid close to $x_{c}^{\alpha \gamma}$, and hence for evaluating velocity at the surface of the particles in the immediate vicinity of the gap, the coefficients that appear in the Lamb's solution are evaluated correctly when $\theta_{0}$ is small.

When two particles with a gap width of $\epsilon$ move with a relative normal velocity of $O(1)$, the lubrication forces in the direction of the separation vector are $O\left(\epsilon^{-1}\right)$. Since these forces are separated by an $O(\epsilon)$ distance, we see from (7) that $A_{j k}^{\alpha \gamma}$ is $O(1)$. In other words, in the outer regionfar from the gap-the lubrication effect appears as a force dipole of an $O(1)$ strength. This also guarantees that the strengths of the multipoles for each particle are also $O(1)$, and hence small compared to the leading order lubrication forces as $\epsilon \rightarrow 0$.

We now proceed to give detailed expressions for $A_{j k}$.

\section{A. Calculation of $\boldsymbol{A}_{j k}^{\alpha \gamma}$}

In this subsection, we shall drop the superscripts $\alpha \gamma$ from various quantities related to the pair $\alpha \gamma$ for clarity. Let us denote the relative motion between the surface of the two particles by

$$
\Delta \mathbf{U} \equiv \Delta U_{m} \mathbf{m}+\Delta U_{t} \mathbf{t}=\left(\mathbf{U}^{\gamma}-\mathbf{\Omega}^{\gamma} \times \mathbf{m}\right)-\left(\mathbf{U}^{\alpha}+\mathbf{\Omega}^{\alpha} \times \mathbf{m}\right),
$$

where $\mathbf{U}$ and $\mathbf{\Omega}$ are, respectively, the translational and rotational velocities, $m$ the unit vector along $x^{\gamma}-x^{\alpha}$, and $t$ the unit vector along the relative tangential velocity in the plane normal to $\mathbf{m}$. The radius of each particle is taken to be unity. Let us choose the cylindrical coordinate system with its axis aligned along $\mathbf{m}$ and with $\mathbf{x}_{c}$ as its origin (cf. 

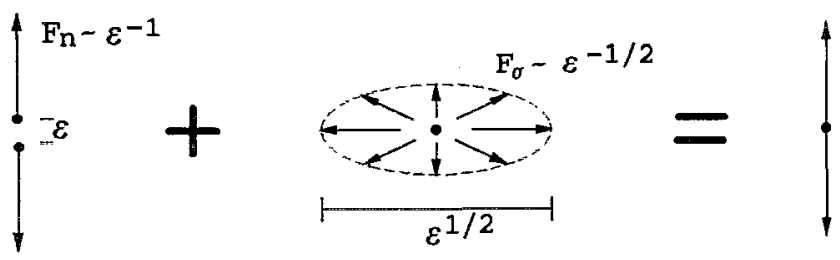

o(1) Force dipole

FIG. 2. A diagrammatic representation of the lubrication velocity field for spheres. Two normal forces of overall magnitude of $O\left(\epsilon^{-1}\right)$ and radial forces of $O\left(\epsilon^{-1 / 2}\right)$ combine to produce the velocity field equivalent to that caused by an $O(1)$ force dipole aligned along the line joining the center of particles.

Fig. 1). Denoting the scaled axial and radial coordinates by $z$ and $\sigma$, with $z=z^{\prime} / \epsilon$ and $\sigma=\sigma^{\prime} / \epsilon^{1 / 2}\left(z^{\prime}\right.$ and $\sigma^{\prime}$ being the unscaled variables), we note that the surface of the particles $\alpha$ and $\gamma$ are approximated by

$$
z=z^{\gamma}=-z^{\alpha}=\left[h+\frac{\epsilon}{2}(h-1)^{2}+O\left(\epsilon^{2}\right)\right], \quad h=1+\frac{\sigma^{2}}{2},
$$

where $\epsilon$ is one-half the minimum gap width. Lubrication forces have been evaluated by several investigators in the past (e.g. Refs. 9-12); taking results from Nunan and Keller, ${ }^{9}$ we have

$$
\begin{aligned}
& \mathbf{f}^{\gamma}=\eta\left[\Delta U_{m}\left(-g_{1} \mathbf{m}-\epsilon^{1 / 2} \sigma g_{2} \mathbf{e}_{\sigma}\right) \epsilon^{-2}-\Delta U_{t} g_{3} \mathbf{t} \epsilon^{-1}\right], \\
& \mathbf{f}^{\alpha}=\eta\left[\Delta U_{m}\left(g_{1} \mathbf{m}-\epsilon^{1 / 2} \sigma g_{2} \mathbf{e}_{\sigma}\right) \epsilon^{-2}+\Delta U_{t} g_{3} \mathbf{t} \epsilon^{-1}\right],
\end{aligned}
$$

where $\mathbf{e}_{\sigma}$ is a unit radial vector in the tangent plane, and

$$
\begin{aligned}
& g_{1}=\frac{3}{8} h^{-2}+\epsilon\left[\frac{3}{10} h^{-1}+\frac{15}{4} h^{-2}-\frac{3}{8} h^{-3}\right]+O\left(\epsilon^{2}\right), \\
& g_{2}=\frac{3}{3} h^{-2}+\epsilon\left[-\frac{3}{4} h^{-1}+\frac{33}{40} h^{-2}\right]+O\left(\epsilon^{2}\right), \\
& g_{3}=\frac{1}{2 z^{\gamma}}+O(\epsilon) .
\end{aligned}
$$

In writing the above expressions for $g_{1}-g_{3}$, we have replaced $\gamma_{i j} n_{i} n_{j} L$ and $\gamma_{i j} n_{i} t_{j} L$ in Nunan and Keller's expressions (B10) $-(\mathrm{B} 14)$ by $\Delta U_{m}$ and $\Delta U_{t}$, respectively. Also, since their $h$ is nondimensionalized with a half-separation length $L=1+\epsilon, h$ in their expressions was replaced by $h(1+\epsilon)$ to obtain the expressions given above.

As mentioned earlier, we shall take $\mathrm{f}^{\mathrm{lub}}$ to be that given by (10)-(11) for $0<\theta<\theta_{0}$ and zero otherwise, $\theta$ being the polar angle measured from $\mathbf{m}$ and $\theta_{0}$ a suitably chosen small number $\left(\epsilon^{1 / 2}<\theta_{0}<1\right)$. The lubrication force on the particles is obtained by integrating (10) or (11) over this range of $\theta$. Using $\sin \theta d \theta=\epsilon d z^{\gamma}$, we obtain

$$
\begin{aligned}
\mathbf{F}^{\gamma}=-\mathbf{F}^{\alpha} & =2 \pi \eta \epsilon^{-1} \int_{1}^{z_{0}}\left[-\Delta U_{m} g_{1} \mathbf{m}-\epsilon \Delta U_{t} g_{3} \mathbf{t}\right] d z^{\gamma} \\
& =-2 \pi \eta\left[\beta_{m} \Delta U_{m} \mathbf{m}+\beta_{t} \Delta U_{t} \mathbf{t}\right]
\end{aligned}
$$

where

$$
\begin{aligned}
& z_{0}=1+2 \epsilon^{-1} \sin ^{2}\left(\theta_{0} / 2\right), \\
& \beta_{t}=\frac{1}{2} \log z_{0},
\end{aligned}
$$

$$
\beta_{m}=\frac{3}{8}\left(1-z_{0}^{-1}\right) \epsilon^{-1}+\frac{27}{40} \log z_{0}+3\left(1-z_{0}^{-1}\right) .
$$

The lubrication force given by (15) agrees with the well known results ${ }^{9-12}$ to $O(\log \epsilon)$ in the limit of $\epsilon \rightarrow 0$ keeping $\epsilon z_{0}$ fixed.

Note that the term containing $g_{2}$ vanished upon integration over the azimuthal angle $\varphi$ in the tangent plane, and thus did not contribute to the lubrication force on the particles. Its contribution to $\mathbf{A}$, however, is nonzero. Substituting (10)-(11) into (7) we obtain

$$
\begin{aligned}
\mathbf{A}= & \frac{1}{4 \pi} \int_{\varphi=0}^{2 \pi} \int_{1}^{z_{0}} d \varphi t d z^{\gamma}\left[\left(\Delta U_{m}\left(g_{1} \mathbf{m}-\epsilon^{1 / 2} \sigma g_{2} \mathbf{e}_{\sigma}\right)\right.\right. \\
& \left.+\Delta U_{g_{3}} \mathbf{t}\right)\left(-z^{\gamma} \mathbf{m}+\epsilon^{-1 / 2} \sigma \mathbf{e}_{\sigma}\right)+\left(\Delta U _ { m } \left(-g_{1} \mathbf{m}\right.\right. \\
& \left.\left.\left.-\epsilon^{1 / 2} \sigma g_{2} \mathbf{e}_{\sigma}\right)-\Delta U_{t} g_{3} \mathbf{t}\right)\left(z^{\gamma} \mathbf{m}+\epsilon^{-1 / 2} \sigma \mathbf{e}_{\sigma}\right)\right] .
\end{aligned}
$$

Integration of $\mathbf{e}_{\sigma} \mathbf{e}_{\sigma}$ with $\varphi$ gives $\pi(\delta-\mathbf{m m}), \delta$ being the Kronecker delta tensor, so that the above expression yields

$$
A_{j k}-\frac{1}{3} \delta_{j k} A_{i i}=\lambda_{m} \Delta U_{m}\left(m_{j} m_{k}-\frac{1}{3} \delta_{j k}\right)+\lambda_{t} \Delta U_{t} t_{j} m_{k},
$$

with

$$
\begin{aligned}
\lambda_{m}= & -\frac{3}{8}\left(1-z_{0}^{-1}\right)-\epsilon\left[\frac{99}{80}\left(z_{0}-1\right)+\frac{87}{40} \log z_{0}\right. \\
& \left.-\frac{9}{80}\left(1-z_{0}^{-1}\right)+\frac{3}{16}\left(1-z_{0}^{-2}\right)\right], \\
\lambda_{t}= & \frac{1}{2} \epsilon\left(1-z_{0}\right) .
\end{aligned}
$$

Note that since $v_{i j}$ is solenoidal, subtracting $1 / 3 \delta_{j k} A_{i i}$ from $A_{j k}$ does not alter $u_{i}^{\text {lub }}$.

Summarizing the above calculations, we see that for normal relative motion $\left(\Delta U_{m} \neq 0\right)$, the normal force density is $O\left(\epsilon^{-2}\right)$ and the area over which it acts is $O(\epsilon)$. This results in an $O\left(\epsilon^{-1}\right)$ normal force on the two particles. These forces are separated by an $O(\epsilon)$ distance and the lubrication velocity field therefore corresponds to that due to an $O(1)$ force dipole aligned along the separation vector. Compared to this, the radial force density is smaller, of $O\left(\epsilon^{-3 / 2}\right)$, but separated by a relatively larger, $O\left(\epsilon^{1 / 2}\right)$, distance, so that the radial motion induces a force dipole of $O\left(\epsilon^{-1}\right)$ in the radial direction. An integration of the flow due to these force dipoles in the tangent plane subsequently results in an overall $O(1)$ force dipole in the direction of $\mathbf{m}$. This is schematically represented in Fig. 2. 
Note that in the limit $\epsilon \rightarrow 0$, keeping $\theta_{0}$ fixed, $\lambda_{m} \rightarrow-3 / 8-99 / 40 \sin ^{2}\left(\theta_{0} / 2\right)$ and $\lambda_{t} \rightarrow-2 \sin ^{2}\left(\theta_{0} / 2\right)$. The lubrication field thus explicitly depends on the cutoff $\theta_{0}$ even though the leading order lubrication force is independent of $\theta_{0}$.

To include the lubrication effects in the numerical scheme presented in I, we modify (19) in I to

$$
\begin{aligned}
u_{i}(\mathbf{x})= & u_{i}^{\infty}(\mathbf{x})+\sum_{\alpha=1}^{N}\left\{\mathscr{G}_{j}^{\alpha} v_{i j}\left(\mathbf{x}-\mathbf{x}^{\alpha}\right)\right. \\
& \left.+\mathscr{H}^{\alpha} \partial_{j} S_{1}\left(\mathbf{x}-\mathbf{x}^{\alpha}\right)\right\}+u_{i}^{\text {lub }}(\mathbf{x}),
\end{aligned}
$$

with

$$
u_{i}^{\mathrm{lub}}(\mathbf{x})=\sum_{\alpha \gamma} \mathscr{G}_{j}^{\alpha \gamma, \mathrm{lub}} v_{i j}\left(\mathbf{x}-\mathbf{x}_{c}^{\alpha \gamma}\right)
$$

where $\mathscr{G}_{j}^{\alpha \gamma \text {,lub }} \equiv\left(A_{j k}^{\alpha \gamma}-1 / 3 \delta_{j k} A_{i i}^{\alpha \gamma}\right) \partial_{k}$.

The contributions to the regular parts of $p_{n}, \chi_{n}$, and $\phi_{n}$ that appear in the Lamb's solution [cf. (6) in I] can be evaluated by expressing the above lubrication differential operator as

$$
\mathscr{G}_{j}^{\alpha \gamma, \mathrm{lub}}=\mathscr{G}_{j}^{\alpha \gamma, P}+\underset{j}{\alpha \gamma, T},
$$

with $\mathscr{G}_{j}^{\alpha \gamma, P}$ and $\mathscr{F}_{j}^{\alpha \gamma, T}$ defined in the same manner as $\mathscr{G}_{j}^{\alpha, P}$ and $\mathscr{F}_{j}^{\alpha, T}$ [cf. (24)-(26), (30)-(32) in I] except that superscript $\alpha$ in those expressions is now replaced by the lubrication pair $\alpha \gamma$. This requires

$P_{20}^{\alpha \gamma}=-3 A_{11}^{\alpha \gamma}, \quad P_{21}^{\alpha \gamma}=A_{12}^{\alpha \gamma}+A_{21}^{\alpha \gamma}, \quad \widetilde{P}_{21}^{\alpha \gamma}=A_{13}^{\alpha \gamma}+A_{31}^{\alpha \gamma}$,

$P_{22}^{\alpha \gamma}=\left(A_{33}^{\alpha \gamma}-A_{22}^{\alpha \gamma}\right) / 2, \quad \tilde{P}_{22}^{\alpha \gamma}=\left(A_{23}^{\alpha \gamma}+A_{32}^{\alpha \gamma}\right) / 2$,

$2 T_{10}^{\alpha \gamma}=A_{23}^{\alpha \gamma}-A_{32}^{\alpha \gamma}, \quad 2 T_{11}^{\alpha \gamma}=A_{13}^{\alpha \gamma}-A_{31}^{\alpha \gamma}, \quad 2 \widetilde{T}_{11}^{\alpha \gamma}=A_{21}^{\alpha \gamma}-A_{12}^{\alpha \gamma}$,

all other $P_{n m}^{\alpha \gamma}$, etc., being zero.

Finally, it is relatively straightforward to evaluate the lubrication force density contribution to the stresslet and torque on each particle. Thus, for example,

$$
\begin{aligned}
\Lambda_{i j}^{\alpha, \mathrm{lub}} & \equiv \int_{S^{\alpha}}\left(x_{i}-x_{i}^{\alpha}\right) f_{j}^{\mathrm{lub}} d A \\
& =\int_{S^{\alpha}}\left[m_{i}(1+\epsilon)+\left(\mathrm{x}-\mathbf{x}_{c}^{\alpha \gamma}\right)_{i}\right] f_{j}^{\mathrm{lub}} d A .
\end{aligned}
$$

The first term in the right-hand side is related to the lubrication force and the second to $A_{i j}$, thus yielding

$$
\Lambda_{i j}^{\alpha, \text { lub }}=(1+\epsilon) m_{i} F_{j}^{\alpha, \text { lub }}+2 \pi \eta A_{j i} \text {. }
$$

\section{B. Choice of $\theta_{0}$}

In order to apply the method described in the previous section to dynamic simulations of suspensions, we need to specify the value of $\theta_{0}$, the polar angle over which the lubrication force density is taken to be nonzero. In addition, we must also specify $\epsilon_{m}$, the maximum value of $\epsilon$ for a pair of particles to qualify as the lubrication pair.

It can be shown that the next term in the Taylor series expansion (5) corresponding to the force quadrupole vanishes, and hence the error in truncating the lubrication field to a force dipole (6) corresponds to that due to a force octupole of magnitude $O\left(\theta_{0}^{2}\right)$. Error introduced in the cal- culations of mobility of a test particle at a distance $R$ from the gap depends not only on $\theta_{0}$ but also on $N_{s}$. Specifically, the contribution to the equation for a $2^{n-1}$-multipole coefficient (e.g. $P_{n m}$ in I) from this neglected octupole at the center of the gap will be $O\left(N_{s}^{2} \theta_{0}^{2} / R^{2}\right)$ compared to that due to an $O(1)$ force dipole at the gap center. Thus, our approximation will fail for a fixed $\epsilon$ and $\theta_{0}$ at sufficiently large $N_{s}$. Fortunately, as $N_{s}$ increases, the regular multipole expansion method usually converges for an increasing number of particle pairs, and the lubrication force density is needed only for particle pairs with $\epsilon<\epsilon_{m}$ with $\epsilon_{m} \rightarrow 0$. Thus, a systematic improvement of the overall mobility tensor with increasing $N_{s}$ will be obtained provided that we decrease $\epsilon_{m}$ with increasing $N_{s}$. In the limit of large $N_{s}$, of course, no lubrication field is necessary, and the current method reduces to the regular multipole expansion method.

Our interest in dynamic simulations, however, is in obtaining reasonably accurate estimates with a small $N_{s}$, e.g., $N_{s}=2$ or 3 . Thus, we shall choose $\theta_{0}$ and $\epsilon_{m}$ somewhat empirically by comparing the results obtained by the present method with small $N_{s}$ against a few test cases for which either the accurate estimates of mobility, etc., are known from previous investigations or can be determined with a fair degree of confidence using the method of multipole expansion described in I.

The first test case we consider is that of determining the effective viscosity of a periodic suspension of rigid spheres defined via ${ }^{9}$

$$
\left\langle d_{i j}\right\rangle \equiv\left\langle\sigma_{i j}-\frac{1}{3} \delta_{i j} \sigma_{k k}\right\rangle=2 \mu_{i j k l}^{*}\left\langle e_{k l}\right\rangle,
$$

where $d_{i j}$ is the deviatoric stress tensor, $e_{k l}=\left(\partial_{l} u_{k}+\partial_{k} u_{l}\right) / 2$ the rate of strain tensor, the angular brackets denote volume averaging over the unit cell, and

$$
\begin{aligned}
\mu_{i j k l}^{*}= & \frac{1}{2} \eta\left[\delta_{i k} \delta_{j l}+\delta_{i l} \delta_{j k}-\frac{2}{3} \delta_{i j} \delta_{k l}\right] \mu_{1}^{*}+\eta\left[\delta_{i j k l}-\frac{1}{3} \delta_{i j} \delta_{k l}\right] \\
& \times\left(\mu_{1}^{*}-\mu_{2}^{*}\right),
\end{aligned}
$$

$\eta$ being the viscosity of the fluid. The nondimensional viscosity coefficients $\mu_{1}^{*}$ and $\mu_{2}^{*}$ are related to $\alpha$ and $\beta$ in Refs. 9 and 13 by $\mu_{1}^{*}=1+\beta$ and $\mu_{2}^{*}=1+\alpha$. The principal lattice directions are taken to coincide with the coordinate axes, and $\delta_{i j k l}=1$ when the indices $i, j, k, l$ are all equal and zero otherwise. Note that such arrays are anisotropic, with $\mu_{2}^{*}-\mu_{1}^{*}$ representing the effect of anisotropy. The coefficients $\mu_{1}^{*}$ and $\mu_{2}^{*}$ as a function of volume concentration $c$ of particles were determined previously by Nunan and Keller ${ }^{9}$ and Sangani and $\mathrm{Lu}^{13}$ In particular, as $c$ approaches its maximum allowable value $c_{\max }$ for the three periodic arrays corresponding to the close packing, the coefficients $\mu_{1}^{*}$ and $\mu_{2}^{*}$ have the limiting behavior for $\tilde{\epsilon} \equiv \epsilon /(1+\epsilon)=1-\left(c / c_{\max }\right)^{1 / 3} \rightarrow 0$ as given by

$$
\mu_{i}^{*}=A_{i} \widetilde{\epsilon}^{-1}+B_{i} \log \widetilde{\epsilon}+C_{i}+O(\widetilde{\epsilon} \log \widetilde{\epsilon}), \quad i=1,2,
$$

with constants $A_{i}-C_{i}$ depending on the geometry of the array. Constants $A_{i}$ and $B_{i}$ were determined by Nunan and Keller from the lubrication analysis, and $C_{i}$ from matching 


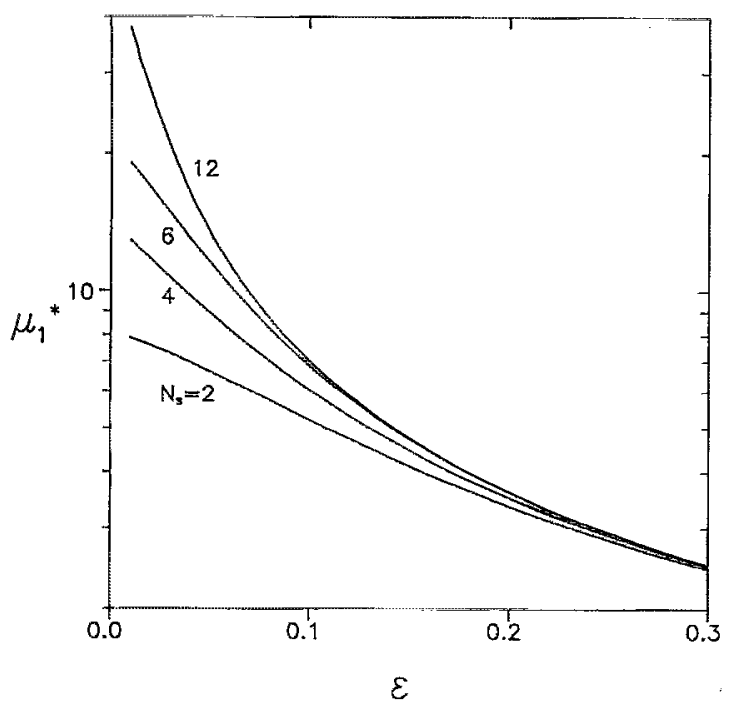

FIG. 3. The viscosity coefficient $\mu_{1}^{*}$ for the face-centered cubic array as a function of $\epsilon$ for various $N_{s}$ using the regular multipole expansion method.

the numerical results for small $\epsilon$ with that given by (31). (Some of the values of $C_{i}$ reported in Ref. 9 had sign errors as pointed out in Ref. 13.)

For a face-centered cubic array, $\mu_{1}^{*}>\mu_{2}^{*}$, and therefore we present in Fig. 3 the results for the former as a function of $\epsilon$ obtained by using the regular multipole expansion method for various $N_{s}$. Results obtained with $N_{s}=12$ may be considered to be exact for the range of $\epsilon$ shown in the figure. These results are in excellent agreement with those in Refs. 9 and 13 and with asymptotic expressions given by (31). The purpose of this figure is to determine $\epsilon_{m}$ as a function of $N_{s}$. Specifically, we choose $\epsilon_{m}$ as that $\epsilon$ for which the difference between the exact and the calculated value of $\mu_{1}^{*}$ for given $N_{s}$ is about $5 \%$. Similar calculations were made for the other two periodic arrays, and it was found that the values of $\epsilon_{m}$ for the three arrays are approximately the same.

The error due to the Taylor series approximation (5) can be compensated against that due to the use of finite $N_{s}$ for some $\theta_{0}$ for each of the three arrays so that it is possible to get exactly the same limiting behavior as (31) for each $N_{s}$. In determining these estimates, which are given in Table I, we used the greater of $\mu_{1}^{*}$ and $\mu_{2}^{*}$ for each array. The estimates of $\theta_{0}$ for odd $N_{s}$ are the same as those for even $N_{s}$ due to the symmetry properties of the periodic arrays. Calculations were next repeated for several different $\epsilon$ values in the range $0.03-\epsilon_{m}$, and the estimates of

TABLE I. $\theta_{0}$ as a function of $N_{s}$ for the three cubic arrays and a pair of particles.

\begin{tabular}{lllll}
\hline \hline$N_{s}$ & SC & BCC & FCC & Pair \\
\hline 2,3 & 0.24 & 0.26 & 0.29 & $0.34,0.26$ \\
4,5 & 0.21 & 0.18 & 0.19 & $0.21,0.18$ \\
6,7 & 0.14 & 0.12 & 0.15 & $0.15,0.13$ \\
\hline
\end{tabular}

TABLE II. A comparison of the results for the effective viscosity $\eta^{*} / \eta$ of random arrays obtained by the present method with those obtained by Ladd.

\begin{tabular}{ccccccc}
\hline \hline & \multicolumn{3}{c}{0.25} & & \multicolumn{2}{c}{0.45} \\
\cline { 2 - 3 } \cline { 5 - 6 }$N_{s} \backslash c$ & Present & Ladd & & Present & Ladd \\
\hline 2 & 2.167 & 2.169 & & 5.41 & 5.41 \\
3 & 2.187 & 2.160 & & 5.43 & 5.29 \\
4 & 2.203 & 2.168 & & 5.46 & 5.48 \\
5 & 2.210 & 2.168 & & 5.51 & 5.41 \\
6 & - & - & & - & 5.54 \\
\hline \hline
\end{tabular}

both $\mu_{1}^{*}$ and $\mu_{2}^{*}$ obtained with the $\theta_{0}$ values given in Table I agreed within $10 \%$ with the corresponding values reported in Refs. 9 and 13. We note that for $N_{s}=2$ and 3, $\theta_{0}$ varied from about 0.24 for simple cubic array to 0.29 for the face-centered cubic array. For larger $N_{s}, \theta_{0}$ is smaller, and its variation for the three different arrays is smaller.

Next, we considered the case of two particles placed along the $x_{1}$-axis separated by a distance of $2(1+\epsilon)$ in a periodic array with a unit cell of a width of 10 units. The purpose in this test calculation was to estimate $\theta_{0}$ for a situation that is considerably different from the concentrated periodic suspension. The mean flow was taken to be the uniaxial extension with its primary extension along the $x_{1}$-axis. The estimates of the velocity of the particles and their stresslet for $\epsilon=0.03$ were obtained from the multipole expansion method with a large $N_{s}$ and compared with those obtained by the lubrication method to determine $\theta_{0}$ for this situation. Results are also shown in Table I, where we see that $\theta_{0}=0.34$ for $N_{s}=2$, a somewhat higher value than one obtained for the periodic arrays. Once again, we see that $\theta_{0}$ decreases with $N_{s}$, and that the difference among different estimates of $\theta_{0}$ decreases with increasing $N_{s}$. Note that the estimates for odd and even $N_{s}$ are different for this configuration of particles.

We now compare the results for the effective viscosity and sedimentation velocity for random suspensions of spheres obtained using the present method with those obtained by Ladd. ${ }^{3}$ Since the periodic array calculations gave the same values of $\theta_{0}$ for odd and even $N_{s}$ values, we found that the effective properties calculated with the $\theta_{0}$ determined from those calculations showed relatively larger fluctuations with $N_{s}$. The results presented in Tables II and III therefore correspond to those obtained with the $\theta_{0}$ given by the last column in Table I. Both the viscosity and the sedimentation velocity were obtained by averaging over 100 random configurations with $N=16$. The results are seen to be in very good agreement with those obtained by Ladd $^{3}$ who used the method of Brady and Bossis. ${ }^{2}$ It should be noted that while accurate results for the effective viscosity are obtained by both methods with $N_{s}=2$, a higher $N_{s}$ is needed for the sedimentation calculations.

\section{THE METHOD FOR CYLINDRICAL PARTICLES}

The method of multipole expansion can also be used to compute the Stokes flow interactions among infinitely long cylinders. The case of flow through a random array of fixed 
TABLE III. A comparison of the results for the sedimentation velocity $U / U_{0}$ of the random arrays of spheres obtained by the present method with those obtained by Ladd.

\begin{tabular}{cccccc}
\hline & \multicolumn{3}{c}{0.25} & & \multicolumn{2}{c}{0.45} \\
\cline { 2 - 3 } \cline { 5 - 6 }$N_{s} \backslash c$ & Present & Ladd & & Present & Ladd \\
\hline 2 & 0.200 & 0.194 & & 0.1010 & 0.0982 \\
3 & 0.170 & 0.166 & & 0.0491 & 0.0480 \\
4 & 0.166 & 0.163 & & 0.0444 & 0.0439 \\
5 & 0.165 & 0.162 & & 0.0426 & 0.0418 \\
\hline \hline
\end{tabular}

cylinders has been treated earlier by Sangani and Yao. ${ }^{14}$ Recently, there is great interest in dynamic simulations of motion of such particles because of its relevance to the diffusion of protein molecules in a bilipid layer of cell membranes. ${ }^{4-7}$ The interesting feature of this problem is that, in addition to the lubrication effects due to relative normal and tangential motion of particles that are important in the interactions of spherical particles, we must also account for lubrication forces arising from a finite amount of fluid flowing through a narrow gap between the cylinders. Since the magnitude of flow through the gap between the particles is related to the difference in the streamfunction values on the surface of the particles, we shall refer to this as the streamfunction lubrication. In what follows, we outline a method to account for this effect; the lubrication effects for relative motion between particles can be treated in the same manner as for the spherical particles, and this and the application of the method to diffusion in bilipid cell membranes will be addressed in a future work.

When the streamfunction on the surface of two cylinders separated by a minimum gap width of $2 \epsilon$ differs by $\Delta \psi$, the tangential component of the velocity in the gap is $O\left(\Delta \psi \epsilon^{-1}\right)$, and the pressure gradient in the gap is $O\left(\mu \Delta \psi \epsilon^{-3}\right)$. With the gap dimension of $O\left(\epsilon^{1 / 2}\right)$ in the tangent direction, this requires that a pressure drop of $O\left(\epsilon^{-5 / 2}\right)$ occur in the fluid flowing through the gap. A detailed analysis then shows this pressure drop to be given by

$$
\frac{\Delta p}{4 \pi \eta} \equiv \lambda=\frac{9 \Delta \psi}{32 \sqrt{2}} \epsilon^{-5 / 2}\left[1+\frac{11}{20} \epsilon+O\left(\epsilon^{2}\right)\right] .
$$

The analysis for cylindrical particles differs significantly from that for spherical particles because of this pressure drop. The leading order force on the particles results in this case from the uneven pressure distribution at the surface of the particles, and this force must therefore be distributed on the entire surface of the particles and not localized to the small region around the gap as was the case for spheres.

Let the $i$ th neighbor of particle $\alpha$ be denoted by $\alpha_{i}$, $\mathbf{m}_{i}$ the unit vector along the line joining the two particles, and $\theta_{i}$ the polar angle made by this vector with the $x_{1}$-axis (cf. Fig. 4). Then, to a first approximation in the outer region, i.e., away from the region of each narrow gap, the pressure appears to have a jump discontinuity of $O\left(\epsilon^{-5 / 2}\right)$ along the surface of the particles. We therefore choose the lubrication force density on particle $\alpha$ to be $f_{i}^{\text {lub }}=-p^{\text {lub }} n_{i}$ with $p^{\text {lub }}$ satisfying

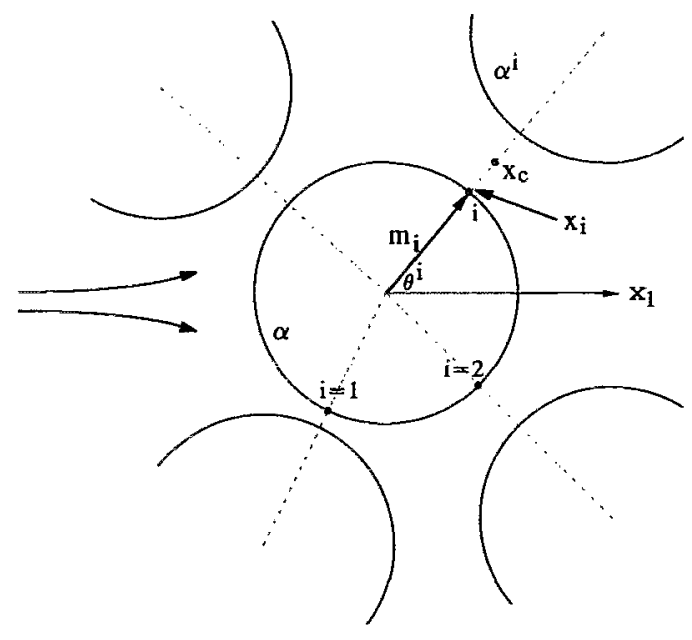

FIG. 4. Nomenclature for the streamfunction lubrication analysis for cylinders.

$$
\frac{1}{4 \pi \eta} \frac{\partial p^{\mathrm{lub}}}{\partial \theta}=\sum_{i} \lambda_{i} \delta\left(\theta-\theta_{i}\right)+g(\theta)
$$

where $\delta$ is Dirac's delta function, $\lambda_{i}$ given by (32), with $\epsilon$ and $\Delta \psi$ in that equation replaced by the corresponding values for the pair $\alpha-\alpha_{i}$, and $g(\theta)$ a regular function to be chosen such that $p^{\text {lub }}$ as defined by (33) is a single-valued function of $\theta$. On integrating (33) over a unit circle, we see that $g(\theta)$ must satisfy

$$
\int_{0}^{2 \pi} g(\theta) d \theta=-\sum_{i} \lambda_{i}
$$

The $x_{1}$-component of the lubrication velocity obtained by substituting $f_{j}^{\mathrm{lub}}=-n_{j} p^{\mathrm{lub}}$ in (3) is given by

$$
u_{1}^{\mathrm{lub}, \alpha}(\mathbf{x})=\frac{1}{4 \pi \eta} \int_{0}^{2 \pi} p^{\mathrm{lub}}(\theta) v_{1 j}(\mathbf{x}-\mathbf{y}) n_{j}(\mathbf{y}) d \theta
$$

with $y_{1}=n_{1}=\cos \theta, y_{2}=n_{2}=\sin \theta$, and $v_{i j}=\delta_{i j} S_{1}-\partial_{i j}^{2} S_{2}$, where $S_{1}$ and $S_{2}$ are now the doubly periodic functions. Now noting that $\partial_{\theta}=\cos \theta \partial_{y_{2}}-\sin \theta \partial_{y_{1}}$ and $\partial_{y_{2}}=-\partial_{x_{2}}$, we have

$$
\begin{aligned}
v_{1 j}(\mathbf{x}-\mathbf{y}) n_{j}(\mathbf{y})= & \cos \theta \frac{\partial^{2} S_{2}}{\partial y_{2}^{2}}(\mathbf{x}-\mathbf{y}) \\
& -\sin \theta \frac{\partial^{2} S_{2}}{\partial y_{1} \partial y_{2}}(\mathbf{x}-\mathbf{y}) \\
= & -\frac{\partial^{2} S_{2}}{\partial x_{2} \partial \theta} S_{2}(\mathbf{x}-\mathbf{y})
\end{aligned}
$$

Using the above result and defining the streamfunction via $u_{1}=\partial \psi / \partial x_{2}$, and $u_{2}=-\partial \psi / \partial x_{1}$, it is easy to show that (35) can be recast to 


$$
\begin{aligned}
\psi^{\mathrm{lub}, \alpha}(\mathbf{x}) & =-\frac{1}{4 \pi \eta} \int_{0}^{2 \pi} p^{\mathrm{lub}}(\theta) \frac{\partial S_{2}}{\partial \theta} d \theta \\
& =\sum_{i} \lambda_{i} S_{2}\left(\mathbf{x}-\mathbf{x}_{i}\right)+\int_{0}^{2 \pi} S_{2}(\mathbf{x}-\mathbf{y}) g(\theta) d \theta
\end{aligned}
$$

where in obtaining the last equality we integrated by parts and made use of (33). Here, $\mathbf{x}_{i}$ is the point on the surface of particle $\alpha$ closest to particle $\alpha_{i}$, i.e. $\mathbf{x}_{i}=\mathbf{x}^{\alpha}+\mathbf{m}_{i}$ (cf. Fig. 4). Now $g(\theta)$ can be expanded in a Fourier series

$$
g(\theta)=g_{0}+\sum_{n=1}^{\infty}\left[g_{n} \cos n \theta+\widetilde{g}_{n} \sin n \theta\right],
$$

and the integral on the extreme right side of (37) can be integrated termwise after substituting for $g(\theta)$. All the resulting terms can be absorbed into the regular multipole expansion at the center of particle $\alpha$ except for the term containing $g_{0}$. On using (34) to evaluate $g_{0}$, and omitting the terms from the other Fourier components, we obtain

$$
\psi^{\mathrm{lub}, \alpha}(\mathbf{x})=\sum_{i} \lambda_{i}\left[S_{2}\left(\mathbf{x}-\mathbf{x}_{i}\right)-S_{2}\left(\mathbf{x}-\mathbf{x}^{\alpha}\right)\right]
$$

The singular part of $S_{2}(\mathbf{r})$ is $r^{2}(1-\log r) / 2$, and the polar components of velocity due to this singularity are $u_{r} \equiv 0$ and $u_{\theta}=-\partial S_{2} / \partial r=r \log r-r / 2$. In other words, this singularity induces a purely swirling flow. The corresponding pressure gradient components are $\partial p / \partial_{r} \equiv 0$ and $\partial p / \partial_{\theta}=-2 \eta$. Thus, the pressure jump discontinuities in the outer region give rise to swirling flow singularities.

There is one further modification necessary to obtain the correct lubrication field. To see this, we note that $\psi^{\text {lub, } \alpha_{i}}$ will have an antisingularity (i.e., of opposite sign) of $O\left(\epsilon^{-5 / 2}\right)$ located at the point $\mathbf{x}^{\alpha}+(1+2 \epsilon) \mathbf{m}_{i}$. For small $\epsilon$ the two singularities cancel to leading order to produce an $O\left(\epsilon^{-3 / 2}\right)$ term given by

$2 \epsilon \lambda_{i}\left[\mathbf{m}_{i} \cdot \nabla+\epsilon^{2} \frac{1}{3 !} \mathbf{m}_{i} \mathbf{m}_{i} \mathbf{m}_{i}(\cdot)^{3} \nabla \nabla \nabla+O\left(\epsilon^{4}\right)\right] S_{2}\left(\mathbf{x}-\mathbf{x}_{c}\right)$,

where $\mathbf{x}_{c}$ is a point midway between particle $\alpha$ and its $i$ th neighbor $\alpha_{i}$ and $(\cdot)^{3}$ stands for a triple scalar product. Now in the narrow gap between these two particles the force density differs from that assumed in writing (33) by an $O\left(\epsilon^{-5 / 2}\right)$ quantity, and hence the lubrication field due to this excess force density may become comparable to that given by (40). Thus, we need to carry out further the analysis of forces acting in the narrow gap between the two particles.

Let us choose the local coordinate axes with the $z$-axis along $\mathbf{m}_{i}$ and the $x$-axis along the tangent direction, denoted by the unit vector $t$. In the following calculations, which refer to the pair $\alpha-\alpha_{i}$, we temporarily suppress the subscript $i$ in $\mathbf{m}_{i}$ for brevity. The origin of the local coordinate axes is $\mathbf{x}_{c}=\mathbf{x}^{\alpha}+(1+\epsilon) \mathbf{m}$. The excess force density on particle $\alpha$ for $x>0$ is evaluated from

$$
\begin{aligned}
\mathbf{f}^{\mathrm{x}} \cdot \mathrm{t} & \equiv f_{x}^{\mathrm{ex}} \\
& =-\left(p-p^{\infty}\right) n_{x}+2 \eta \partial_{x y}^{2} \psi n_{x}+\eta\left(\partial_{z z}^{2}-\partial_{x x}^{2}\right) \psi n_{z} .
\end{aligned}
$$

A similar expression can be written for $f_{z}^{\mathrm{ex}}$. Here $p^{\infty}$ is the pressure in the fluid just outside the gap $(x>0)$, beyond which the pressure gradient in the fluid becomes $O(1)$. The reason for subtracting this quantity from the actual force density is that our previous calculation, which resulted in (39), has already accounted for the pressure distribution outside the gap via (33). The expression for $f_{x}$ for $x<0$ is obtained by substituting $p^{-\infty}$ in lieu of $p^{\infty}$ in (41). Note that $p^{-\infty}-p^{\infty}=\Delta p=4 \pi \eta \lambda_{i}$ [cf. (32)]. The lubrication field due to this excess force density is

$$
\begin{aligned}
\psi^{\mathrm{ex}}(\mathbf{x})= & -(4 \pi \eta)^{-1} \int\left[f_{x}^{\mathrm{ex}}(\mathbf{y}) \mathrm{m}-f_{z}^{\mathrm{ex}}(\mathbf{y}) \mathrm{t}\right] \\
& \cdot \nabla S_{2}(\mathbf{x}-\mathbf{y}) d A_{\mathrm{y}},
\end{aligned}
$$

where the integration is to be carried over the portions of both particles, $\alpha$ and $\alpha_{i}$ over which this excess force density remains large in magnitude and $\mathbf{y}$ is the dummy variable of integration. It is easy to show that

$$
f_{x}^{\mathrm{ex}, \alpha}(x)=f_{x}^{\mathrm{ex}, \alpha_{i}}(x)=f_{x}^{\mathrm{ex}, \alpha}(-x),
$$

and

$$
f_{z}^{\mathrm{ex}, \alpha}(x)=-f_{z}^{\mathrm{ex}, \alpha_{i}}(x)=-f_{z}^{\mathrm{ex}, \alpha}(-x) .
$$

Now the integral in (42) can be evaluated approximately using the Taylor series expansion of $S_{2}(x-y)$ around $S_{2}\left(\mathrm{x}-\mathrm{x}_{c}\right)$ and integrating each term separately as in the case of spherical particles. To leading orders, $f_{z}=O\left(\epsilon^{-5 / 2}\right)$ and $f_{x}=O\left(\epsilon^{-2}\right)$. However, because of the asymmetric properties of $f_{z}$, the leading order contribution to $\psi^{\text {lub,ex }}$ arises from $f_{x}$, and this $O\left(\epsilon^{-3 / 2}\right)$ term upon detailed calculations was found to balance exactly the leading term in (40). This makes it necessary to evaluate the next correction of $O\left(\epsilon^{-1}\right)$ to $f_{x}$. At this next order, there is also a contribution from $f_{z}$ so that on making use of the Taylor series expansion we obtain

$$
\begin{aligned}
\psi^{\mathrm{lub}, \mathrm{ex}} \simeq & -\frac{1}{2 \pi \eta} \int_{\varphi>0} d \varphi\left[2 f_{x}^{\mathrm{ex}, \alpha} m_{j} \partial_{j}\right. \\
& +\epsilon x\left(x f_{x}^{\mathrm{ex}, \alpha}+2 \epsilon^{1 / 2} h f_{z}^{\mathrm{ex}, \alpha}\right) m_{i} t_{j} t_{k} \partial_{i j k}^{3} \\
& \left.+\epsilon^{2} h^{2} f_{x}^{\mathrm{ex}, \alpha} m_{i} m_{j} m_{k} \partial_{i j k}^{3}\right] S_{2}\left(\mathbf{x}-\mathbf{x}_{c}\right),
\end{aligned}
$$

where $\varphi=\theta_{i}-\theta$ is the polar angle measured from $\mathbf{m}$ and $x$ and $h$ the scaled variables with $x=x^{\prime} / \epsilon^{1 / 2}$ and $h=1+x^{2} / 2$. Also, we have made use of the symmetry properties listed in (43)-(44) to reduce the integration on the surface of both particles to that for $\varphi>0$ on particle $\alpha$. Note that the contribution from $f_{z}$, of $O\left(\epsilon^{-1 / 2}\right)$, is of the same order of magnitude as that from $x^{2} f_{x}$, and that the lubrication fields due to both of them combine to give a force quadrupole at the center of the gap. Once again detailed calculations showed that the leading $O\left(\epsilon^{-1 / 2}\right)$ terms arising from each of these two terms cancel out leaving finally the lubrication streamfunction consisting of $O(1)$ 
force and quadrupoles at the center of the gap. Finally, the last term, viz. $h^{2} f_{x}$, in the above expression is actually small, of $O\left(\epsilon^{1 / 2}\right)$, and could have been omitted from the lubrication field description. However, since this term also gives rise to a force quadrupole and can therefore be included along with the terms from $f_{z}$ and $f_{x} x^{2}$ with very little additional work, we have retained it in the calculations. Now the standard lubrication analysis yields

$$
\begin{aligned}
\begin{array}{l}
\psi= \\
\psi_{0}^{\alpha}
\end{array}+\Delta \psi\left[\frac{3}{4}\left(\frac{z}{h}-\frac{1}{3} \frac{z^{3}}{h^{3}}\right)+\epsilon\left\{\left(\frac{21}{40} h-\frac{3}{5}\right)\left(\frac{z^{5}}{h^{5}}-2 \frac{z^{3}}{h^{3}}+\frac{z}{h}\right)\right.\right. \\
\left.\left.-\frac{3}{8} \frac{(h-1)^{2}}{h}\left(\frac{z}{h}-\frac{z^{3}}{h^{3}}\right)\right\}+O\left(\epsilon^{2}\right)\right] \\
f_{x}^{\mathrm{ex}}=\eta \Delta \psi \epsilon^{-2}\left[-\frac{3}{8 h}+\frac{3}{4 h^{2}}-\frac{9}{16}\left(1+\frac{11}{20} \epsilon\right)(x I-2)\right. \\
\left.+\epsilon\left\{-\frac{177}{160 h}+\frac{294}{160 h^{2}}-\frac{3}{4 h^{3}}\right\}+O\left(\epsilon^{2}\right)\right] \\
f_{z}^{\mathrm{ex}}=\eta \Delta \psi \epsilon^{-5 / 2}\left[-\frac{9}{16} I+\frac{9 x}{16 h}+\frac{3 x}{8 h^{2}}+O(\epsilon)\right]
\end{aligned}
$$

where

$$
I=\sqrt{2}\left[\frac{\pi}{2}-\tan ^{-1}\left(\frac{x}{\sqrt{2}}\right)\right] .
$$

Here, $\psi_{0}^{\alpha}$ is the streamfunction value on particle $\alpha$, and $\Delta \psi=\psi^{\alpha_{i}}-\psi^{\alpha}$. Substituting the above expressions into (45), using $d \varphi=\epsilon^{1 / 2}(1+\epsilon(h-1)) d x$, and carrying out integration from $x=0$ to $x=x_{0}=\varphi_{0} / \epsilon^{1 / 2}$ we obtain

$$
\begin{aligned}
\psi^{\mathrm{lub}, \mathrm{ex}}(\mathbf{x})= & \Delta \psi\left[\beta_{1} m_{j} \partial_{j}+\left(\mathscr{R}_{2} m_{i} t_{j} t_{k}+\beta_{3} m_{i} m_{j} m_{k}\right) \partial_{i j k}^{3}\right] \\
& \times S_{2}\left(\mathbf{x}-\mathbf{x}_{c}\right),
\end{aligned}
$$

where

$$
\begin{aligned}
& \beta_{1}=-\frac{9 \epsilon^{-3 / 2}}{16 \sqrt{2}}\left[1+\frac{11}{20} \epsilon\right]+\mathscr{R}_{1}, \\
& \beta_{3}=-\frac{3 \epsilon^{1 / 2}}{32 \sqrt{2}}+\mathscr{R}_{3},
\end{aligned}
$$

with

$$
\begin{aligned}
\mathscr{R}_{1}= & \frac{9 \epsilon^{-3 / 2}}{16 \pi}\left[\left(1+\frac{11}{20} \epsilon\right)\left(I_{0} h_{0}-x_{0}\right)-\frac{2}{3} x_{0} h_{0}^{-1}\right. \\
& \left.+\epsilon\left\{\frac{1}{8} x_{0}^{4} I_{0}-\frac{x_{0}^{3}}{4}+\frac{x_{0}}{6}-\frac{7}{15} x_{0} h_{0}^{-1}+\frac{1}{3} x_{0} h_{0}^{-2}\right\}\right], \\
\mathscr{K}_{2}= & \frac{3 \epsilon^{-1 / 2}}{32 \pi}\left[\frac{3}{2} x_{0}^{4} I_{0}+3 x_{0}^{2} I_{0}+4 x_{0} h_{0}^{-1}-4 x_{0}-3 x_{0}^{3}\right], \\
\mathscr{R}_{3}= & \frac{3 \epsilon^{1 / 2}}{32 \pi}\left[h_{0}^{3} I_{0}-7 x_{0}-\frac{4}{3} x_{0}^{3}-\frac{1}{4} x_{0}^{5}\right],
\end{aligned}
$$

wherein $I_{0} \equiv I\left(x_{0}\right)$ and $h_{0}=1+x_{0}^{2} / 2$. Note that $\mathscr{R}_{i} \rightarrow 0$ as $x_{0} \rightarrow 0$ and, in the limit $\epsilon \rightarrow 0$ keeping $\varphi_{0}=\epsilon^{1 / 2} x_{0}$ fixed,

$$
\mathscr{R}_{1} \rightarrow \frac{6}{5 \pi \varphi_{0}^{3}}, \quad \mathscr{R}_{2} \rightarrow \frac{3}{5 \pi \varphi_{0}}, \quad \mathscr{R}_{3} \rightarrow-\frac{9 \varphi_{0}}{20 \pi}
$$

Thus, contributions from each of these terms are $O(1)$ for fixed $\varphi_{0}$ as $\epsilon \rightarrow 0$. Now the terms of $O\left(\epsilon^{-3 / 2}\right)$ and $O\left(\epsilon^{-1 / 2}\right)$ from $\beta_{1}$ in (51) exactly cancel the corresponding terms in (40), and the first term on the right-hand side of (52) cancels the second term in (40) leaving contibutions of $O(1)$ arising from the cutoff at finite $\theta_{0}$ in $\mathscr{R}_{i}(i=1,3)$.

In summary, the complete expression for streamfunction is given by

$$
\begin{aligned}
\psi(\mathbf{x})= & \psi^{\infty}(\mathbf{x})+\sum_{\alpha=1}^{N}\left[B_{0}^{\alpha} S_{1}\left(\mathbf{x}-\mathbf{x}^{\alpha}\right)+\sum_{n=1}^{N_{s}}\left\{\left(A_{n}^{\alpha} \partial_{1}\right.\right.\right. \\
& \left.+\widetilde{A}_{n}^{\alpha} \partial_{2}\right) \partial_{1}^{n-1} S_{2}\left(\mathbf{x}-\mathbf{x}^{\alpha}\right)+\left(B_{n}^{\alpha} \partial_{1}+\widetilde{B}_{n}^{\alpha} \partial_{2}\right) \\
& \left.\left.\times \partial_{1}^{n-1} S_{1}\left(\mathbf{x}-\mathbf{x}^{\alpha}\right)\right\}\right]+\psi^{\mathrm{lub}}(\mathbf{x})
\end{aligned}
$$

where $\psi^{\infty}$ is the streamfunction corresponding to the imposed flow, $A_{n}^{\alpha}, B_{n}^{\alpha}$, etc., the coefficients of various multipoles, ${ }^{14}$ and $N_{s}$ the maximum order of multipole retained in solving interactions among particles. The lubrication field consists of two parts:

$$
\begin{aligned}
\psi^{\mathrm{lub}}(\mathbf{x})= & \sum_{\alpha=1}^{N} \sum_{i}-\lambda_{i} S_{2}\left(\mathbf{x}-\mathbf{x}^{\alpha}\right)+\sum_{\alpha \gamma} \Delta \psi^{\alpha \gamma}\left[\mathscr{R}_{1} m_{j} \partial_{j}\right. \\
& \left.+\left(\mathscr{R}_{2} m_{i} t_{j} t_{k}+\mathscr{R}_{3} m_{i} m_{j} m_{k}\right) \partial_{i j k}^{3}\right] S_{2}\left(\mathbf{x}-\mathbf{x}_{c}^{\alpha \gamma}\right),
\end{aligned}
$$

where, in the first part, the summation is over all the neighbors $i$ of particle $\alpha$, and, in the second part, the summation is over all pairs $\alpha \gamma$ with a separation distance less than a specified value, $\mathbf{m}$ is a unit vector along $\mathbf{x}^{\gamma}-\mathbf{x}^{\alpha}, \mathbf{x}_{c}$ is midway between the two particles, and $\Delta \psi^{\alpha \gamma}=\psi^{\gamma}-\psi^{\alpha}$. Since $\lambda_{i}=O\left(\epsilon^{-5 / 2}\right) \Delta \psi_{i}$, the first part of the lubrication field tries to force each cylinder to take up the streamfunction values in such a manner that the summation of $\lambda_{i}$ over all the neighbors of a particle is small and can be balanced with the rest of the $O(1)$ terms in the streamfunction expression. To put it in another way, the first part is responsible for a global allocation of the given flow into the flow through each individual gap. The second part of the lubrication field corresponds to the $O(1)$ force and force quadrupoles at the center of each narrow gap between the particles. The calculations leading to the expression for $\psi^{\text {lub }}$ are diagrammatically summarized in Fig. 5.

The force on particle $\alpha$ is evaluated from

$$
\begin{aligned}
& F_{1}^{\alpha}=4 \pi \eta\left[\tilde{A}_{1}^{\alpha}+\sum_{i}\left\{\left(\lambda-\frac{\beta_{1}}{2}\right) m_{2}\right\}_{i}\right], \\
& F_{2}^{\alpha}=-4 \pi \eta\left[A_{1}^{\alpha}+\sum_{i}\left\{\left(\lambda-\frac{\beta_{1}}{2}\right) m_{1}\right\}_{i}\right],
\end{aligned}
$$

where the subscript $i$ refers to the quantities related to the pair $\alpha-\alpha_{i}$, and $\lambda$ and $\beta_{1}$ are given by (32) and (51), respectively. 

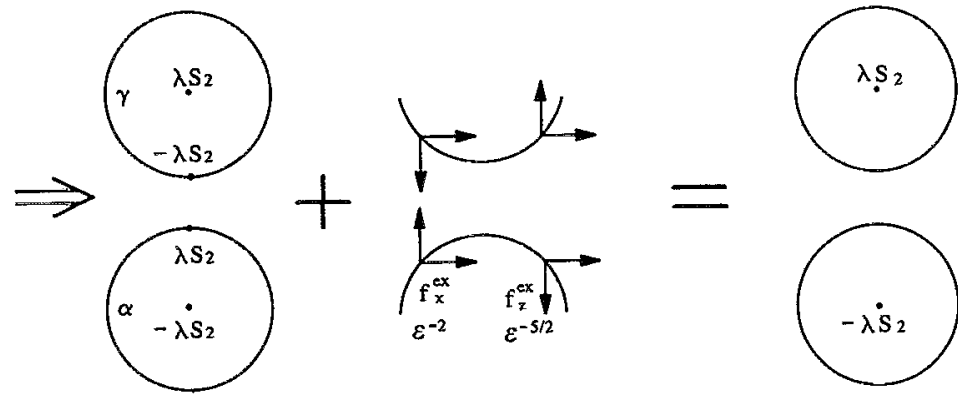

$\lambda \sim\left(\psi^{\gamma}-\psi^{\alpha}\right) \varepsilon^{-5 / 2}$

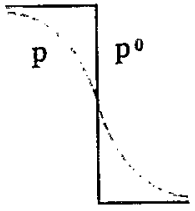

(a)

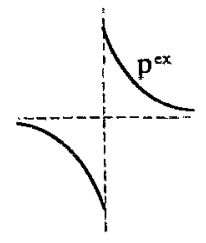

(b)

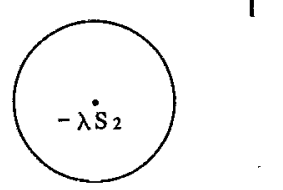

$\underset{\lambda \varepsilon \mathrm{F}^{0}}{\longrightarrow} \underset{\lambda \varepsilon{ }^{3} \mathrm{Q}^{0}}{\longrightarrow}$

$+$

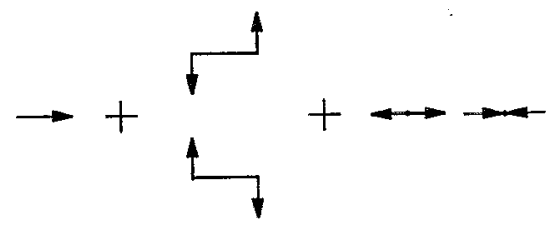

(d)

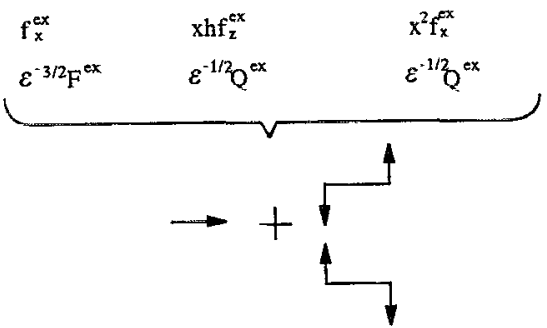

$\mathrm{O}(1) \mathrm{F} \quad \mathrm{O}(1) \mathrm{Q}$ (c)

(e)

FIG. 5. A diagrammatic representation of the streamfunction lubrication field for cylinders. The outer region $O\left(\epsilon^{-5 / 2}\right)$ pressure jump distribution is represented by four swirling flow $\left(S_{2}\right)$ singularities in (a), two of which near the gap combine to produce an $O\left(\epsilon^{-3 / 2}\right)$ force singularity pointing in the direction opposite to the flow and a force quadrupole of $O\left(\epsilon^{1 / 2}\right)$ as shown in (c). The excess force density distribution in the gap shown in (b) combines to an $O\left(\epsilon^{-3 / 2}\right)$ force and $O\left(\epsilon^{-1 / 2}\right)$ force quadrupole singularities as shown in (d). The leading order force in (d) is canceled by the force in (c) and the two quadrupoles in (d) cancel to leading $O\left(\epsilon^{-1 / 2}\right)$ producing thereby a velocity field consisting of two $O\left(\epsilon^{-5 / 2}\right)$ swirling flow singularities at the center of the particles and $O(1)$ force and force quadrupole singularities at the center of the gap between the two particles as shown in (e).

\section{A. Choice of $\varphi_{0}$ and $\epsilon_{m}$}

We used the results for the viscous drag on a fixed periodic array of cylinders to determine $\varphi_{0}$ and $\epsilon_{m}$. First, the drag on a cylinder was determined for various $N_{s}$ and $\epsilon=h / 2-1, h$ being the lattice spacing. For each $N_{s}, \epsilon_{m}$

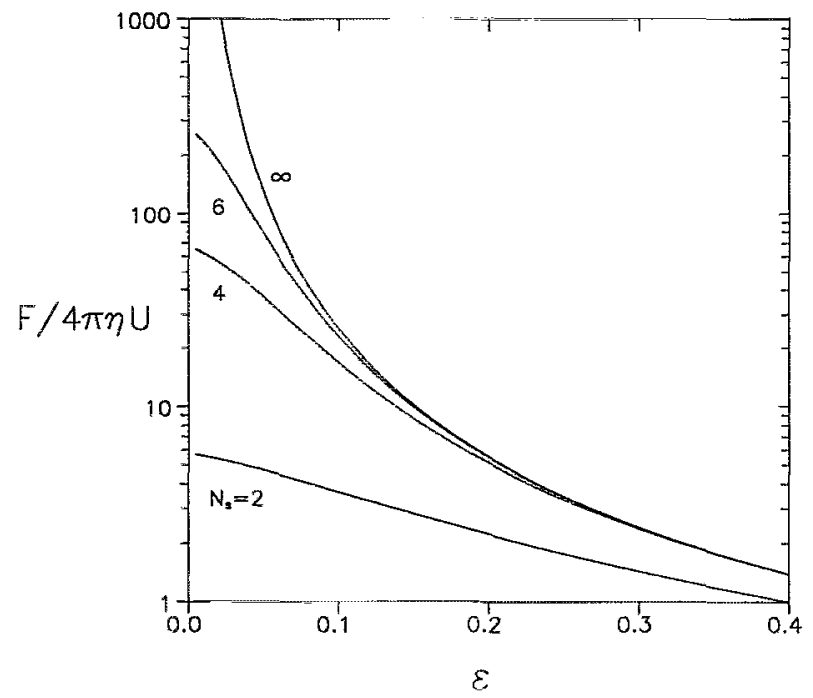

FIG. 6. The nondimensional drag for square array of cylinders for various $N_{s}$ obtained by the regular multipole expansion method. was determined using the criterion that the drag for $\epsilon>\epsilon_{m}$ was accurate to within $5 \%$ without the lubrication field. Figure 6 shows the results for the nondimensional drag $F / 4 \pi \eta U$ for a square array of cylinders obtained using the regular multipole expansion method with different values of $N_{s}$. The exact curve corresponds to $N_{s}=15$. These results are in excellent agreement with those reported by Sangani and Acrivos ${ }^{15}$ who used a boundary collocation method. By comparing the results with different $N_{s}$, we determined $\epsilon_{m}$ to equal 0.4 for $N_{s}=1,2,0.20$ for $N_{s}=3,4,0.15$ for $N_{s}=5,6$, and 0.075 for $N_{s}=7,8$.

TABLE IV. The nondimensional viscous drag $F / 4 \pi \eta U$ as a function of $N_{s}$ for a single configuration of 16 cylinders.

\begin{tabular}{|c|c|c|c|c|}
\hline \multirow[t]{2}{*}{$N_{s} \backslash c$} & \multicolumn{2}{|c|}{0.6} & \multicolumn{2}{|c|}{0.4} \\
\hline & $\begin{array}{c}\text { w/o lub. } \\
37.2\end{array}$ & $\begin{array}{c}\text { with lub. } \\
175.2\end{array}$ & $\begin{array}{c}\text { w/o lub. } \\
7.7\end{array}$ & $\begin{array}{c}\text { with lub. } \\
10.5\end{array}$ \\
\hline 2 & 74.6 & 185.1 & 10.7 & 12.1 \\
\hline 3 & 86.0 & 169.0 & 11.8 & 12.0 \\
\hline 4 & 129.2 & 174.1 & 12.1 & 12.2 \\
\hline 5 & 145.7 & 174.2 & 12.2 & 12.2 \\
\hline 6 & 156.1 & 166.0 & & \\
\hline 8 & 167.3 & & & \\
\hline 10 & 169.3 & & & \\
\hline 12 & 170.3 & & & \\
\hline 14 & 170.7 & & & \\
\hline 15 & 170.8 & & & \\
\hline
\end{tabular}


TABLE $\mathrm{V}$. The nondimensional $\operatorname{drag} F / 4 \pi \eta U$ for random arrays of cylinders with $N=64$.

\begin{tabular}{ccccccc}
\hline \hline$c$ & 0.1 & 0.2 & 0.3 & 0.4 & 0.5 & 0.6 \\
\hline$F / 4 \pi \eta U$ & 1.5 & 3.7 & 8.9 & 22.1 & 60.4 & 219 \\
\hline
\end{tabular}

Next, calculations were made for various $\epsilon$ values smaller than $\epsilon_{m}$ for different $\varphi_{0}$ to determine the optimum values of $\varphi_{0}$ for each $N_{s}$. Results were relatively insensitive to the choice of $\varphi_{0}$, and we estimated $\varphi_{0}$ to equal 0.1 for $N_{s}=1,2,0.25$ for $N_{s}=3-6$, and 0.18 for $N_{s}=7$, 8. With these values of $\varphi_{0}$, the calculated values of the nondimensional drag agreed to within about $5 \%$ for all area concentrations of cylinders for each $N_{s}$.

With $\epsilon_{m}$ and $\varphi_{0}$ chosen as above, we next carried out calculations for random arrays with $N=16$ at various $c$. Only one configuration was chosen for each $c$, and the mean flow velocity components along $x_{1}$ and $x_{2}$-axes were chosen to be unity. Results for the mean drag with $c$, the area fraction of cylinders, equal to 0.6 are given in Table IV. We see that the method is remarkably accurate. The importance of using the lubrication field decreases for smaller $c$. For example, the convergence of the drag values for $c=0.3$ both with and without the lubrication fields are comparable, and thus there is no special advantage in including the lubrication field for $c \leqslant 0.3$.

In Sangani and Yao, ${ }^{14}$ we presented very limited results for the nondimensional drag on cylinders because of the computational limitations then. For example, the calculations there were limited to $N=16$ for $c<0.5$ and to $N=9$ for $c=0.7$. Also, the estimates of the force were obtained by averaging over less than 10 configurations. At high $c$ the degree of anisotropy with small $N$ is very severe and large $N$ is required to obtain good estimates. With the lubrication method outlined here the computational time with $N=64$ is about $2-3 \mathrm{~s}$ on an IBM machine at the Cornell Theory Center. Thus, we have recalculated the results by averaging over 20 configurations for each $c$, and the results are presented in Table $\mathrm{V}$.

\section{ACKNOWLEDGMENTS}

The work was supported by NSF under Grant Nos. CTS-9118675 and CTS-9307723. The use of supercomputer facilities at the Cornell Theory Center is also gratefully acknowledged.

${ }^{\prime}$ G. Mo and A. S. Sangani, "A method for computing Stokes flow interactions among spherical objects and its application to suspensions of drops and porous particles," Phys. Fluids 6, 1637 (1994).

${ }^{2}$ J. F. Brady and G. Bossis, "Stokesian dynamics," Annu. Rev. Fluid Mech. 20, 111 (1988).

${ }^{3}$ A. J. C. Ladd, "Hydrodynamic transport coefficients of random dispersions of hard spheres," J. Chem. Phys. 95, 3484 (1990).

${ }^{4}$ P. G. Saffman and M. Delbruck, "Brownian motion in biological membranes," Proc. Natl. Acad. Sci. USA 72, 3111 (1975).

${ }^{5}$ S. J. Bussell, D. L. Koch, and D. A. Hammer, "The resistivity and mobility functions for a model system of two-equal sized proteins in a lipid bilayer," J. Fluid Mech. 243, 679 (1992).

${ }^{6} \mathrm{~S}$. J. Bussell, D. A. Hammer, and D. L. Koch, "The effect of hydrodynamic interactions on the tracer and gradient diffusion of integral membrane proteins in lipid bilayers," submitted to J. Fluid Mech.

${ }^{7}$ S. J. Bussell, D. L. Koch, and D. A. Hammer, "The effect of hydrodynamic interactions on the diffusion of integral membrane proteins: Diffusion in plasma membranes," submitted to Biophys. J.

${ }^{8}$ A. A. Zick and G. M. Homsy, "Stokes flow through periodic arrays of spheres," J. Fluid Mech. 115, 13 (1982).

${ }^{9} \mathrm{~K}$. C. Nunan and J. B. Keller, "Effective viscosity of a periodic suspension," J. Fluid Mech. 142, 269 (1984).

${ }^{10} \mathrm{~J}$. Happel and H. Brenner, Low Reynolds Number Hydrodynamics (Martinus Nijhoff, Dordrecht, 1973).

"S. Kim and S. J. Karrila, Microhydrodynamics: Principles and Selected Applications (Butterworth-Heinemann, London, 1991).

${ }^{12}$ D. J. Jeffrey and Y. Onishi, "Calculation of the resistance and mobility functions for two unequal rigid spheres in low-Reynolds-number flow," J. Fluid Mech. 139, 261 (1984).

${ }^{13}$ A. S. Sangani and W. Lu, "Effective viscosity of an ordered suspension of small drops," J. Appl. Math. Phys. (ZAMP) 38, 557 (1987).

${ }^{14}$ A. S. Sangani and C. Yao, "Transport processes in random arrays of cylinders. II. Viscous flow," Phys. Fluids 31, 2435 (1988).

${ }^{15}$ A. S. Sangani and A. Acrivos, "Slow flow past periodic arrays of cylinders with application to heat transfer," Int. J. Multiphase Flow 8 , 193 (1982). 